\title{
Teste de caminhada de seis minutos em idosos de uma instituição de longa permanência: valores, aplicabilidade e correlações
}

\author{
Chandra da Silveira Langoni", Alice Carvalho Borsatto", \\ Luiza Armani Valmorbida"*, Thais de Lima Resende ${ }^{*+*}$
}

\section{Resumo}

O sedentarismo, ente outros fatores, conduz à prevalência aumentada de limitações físicas e comorbidades em idosos residentes em instituição de longa permanência (institucionalizados). Assim, determinar meios e/ou instrumentos que auxiliem a detecção e a prevenção de ambas as limitações é fundamental para garantir melhor saúde e qualidade de vida. Dentre os muitos instrumentos utilizados para tal, o teste da caminhada de seis minutos (TC6M) avalia, objetivamente, o grau de capacidade física em indivíduos com condicionamento físico limitado. Assim, o presente estudo foi desenvolvido com os seguintes objetivos: (1) determinar a distância máxima percorrida por idosos institucionalizados no TC6M; (2) verificar a relação entre os resultados do item um e as variáveis sexo, idade e grau de independência funcional; e (3) Estabelecer o nível de segurança da aplicação desse teste na população estudada. Participaram desse estudo transversal 33 idosos (12 mulheres), classificados como independentes e semi-independentes (Índice de Barthel), aptos a realizar o TC6M. Os participantes, com idades entre 71 e 80 anos, tiveram seus sinais vitais mensurados antes e depois do teste. A média da distância máxima percorrida pelos idosos foi de 250 metros, muito inferior à média de 450 metros de idosos com evidência de cardiopatia encontrada na literatura. Não foi detectada diferença significante na comparação das médias em termos de sexo, idade ou grau de independência funcional. Conclui-se que os idosos institucionalizados percorreram distâncias menores do que o esperado, apresentando, portanto, condicionamento físico limitado. O teste de caminhada de seis minutos tem boa aplicabilidade para esse público.

Palavras-chave: Instituições de longa permanência para idosos. Idoso. Envelhecimento. Condicionamento físico humano.

* Fisioterapeuta, Especialista em Residência Multiprofissional em Saúde da Família e da Comunidade pela Pontifícia Universidade Católica do Rio Grande do Sul (PUCRS).

** Fisioterapeuta.

**** Fisioterapeuta, Especialista em Fisioterapia em Geriatria e Gerontologia.

***** Fisioterapeuta. Doutora em Ciências da Saúde. Professora Titular da Faculdade de Enfermagem, Nutrição e Fisioterapia (Faenfi) da PUCRS. PUCRS/Faenfi - Endereço para correspondência: Avenida Ipiranga, n. 6681, prédio 12 A $8^{\circ}$ andar, Partenon, CEP: 90619-900. E-mail: athaislr@gmail.com.

$\rightarrow$ http://dx.doi.org/10.5335/rbceh.2013.3260 


\section{Introdução}

O envelhecimento da população mundial (PAPALÉO; PONTE, 2005), incluindo a população brasileira (BRASIL, 2012), torna imperativas as questões de saúde, de autonomia e de qualidade de vida do idoso. Aliadas a tal envelhecimento, as alterações estruturais e funcionais, embora variem de um indivíduo para outro, são próprias do processo fisiológico de envelhecimento (PAPALÉO; PONTE, 2005) e tornam-se problemas de saúde pública, tendo em vista o rápido crescimento da população idosa.

Cresce, também, o percentual de idosos que dependente de cuidados, o que torna cada vez mais necessárias as instituições que atendem a esses indivíduos (GUCCIONE, 2002). Os diferentes motivos de institucionalização e a falta de alternativas de atenção aos idosos tornam a instituição de longa permanência (ILPI) indispensável e insubstituível no sistema social vigente do país (CREUTZBERG, 2005).

Os idosos residentes em instituição de longa permanência (institucionalizados) apresentam um perfil diferenciado: grandes níveis de sedentarismo, condicionamento físico limitado, carência afetiva, perda de autonomia causada por incapacidades físicas e mentais, ausência de familiares para ajudar no autocuidado e insuficiência de suporte financeiro. Esses fatores contribuem para a grande prevalência de limitações físicas e comorbidades, refletindo, negativamente, em sua independência e autonomia (CREUTZBERG, 2005).

É sabido que a prática de atividade física pode minimizar a maioria das per- das que acompanham o envelhecimento (SUN et al., 2013), melhorando de forma global a saúde do idoso, inclusive no que tange ao risco de quedas (PIMENTEL; SCHEICHER, 2009). Desse modo, tal prática é recomendada, inclusive para idosos institucionalizados, para os quais a associação de sedentarismo, de condicionamento físico limitado, de limitações físicas e de comorbidades torna necessária a realização de uma avaliação criteriosa antes do seu envolvimento em qualquer tipo de atividade física, inclusive aquelas típicas de um tratamento fisioterapêutico (IORIS, 2002). Torna-se fundamental certificar-se de que isso ocorrerá em condições de segurança clínica para o indivíduo cuidado. Portanto, métodos e/ou instrumentos que permitam que a prática de atividade física, por idosos, em quaisquer contextos, seja desenvolvida dentro de limites seguros, são de relevância ímpar.

Dentre os muitos instrumentos utilizados para avaliar a capacidade funcional e emitir um prognóstico clínico de indivíduos com doenças cardíacas e pulmonares, selecionou-se o teste da caminhada de seis minutos - TC6M (AMERICAN THORACIC SOCIETY, 2002). Esse é um método simples, de fácil aplicação e de baixo custo, por isso, muito utilizado para avaliar objetivamente o grau de capacidade física em pacientes com o condicionamento físico limitado (AMERICAN THORACIC SOCIETY, 2002). Por meio desse teste, é possível avaliar a capacidade submáxima de exercício, posto que a distância que um indivíduo percorre em seis minutos reflete melhor as suas atividades físicas 
diárias do que testes máximos de exercício (SOLWAY et al., 2001).

Assim, considerando as características da institucionalização e suas consequências, fica clara a necessidade da determinação da segurança e dos valores do TC6M em idosos institucionalizados, de forma a permitir a prática segura de atividades físicas pela população em questão. $\mathrm{O}$ presente estudo, por conseguinte, foi desenvolvido com os seguintes objetivos: determinar a segurança da aplicação do TC6M em idosos institucionalizados e os valores desse teste na população em estudo, bem como verificar sua relação com sexo, idade e grau de independência funcional.

\section{Métodos}

Este estudo observacional, analítico e transversal utilizou dados coletados do estudo Instituições de longa permanência para idosos - ILPIs no Brasil: tipologia e proposta de modelo básico de assistência multidimensional, desenvolvido no período de dezembro de 2008, em seis diferentes centros no Brasil, entre os quais Porto Alegre. Os dados a serem apresentados foram coletados em uma das instituições participantes, a Sociedade porto-alegrense de auxílio aos necessitados (Spaan), localizada em Porto Alegre, RS/Brasil.

Dentre os 136 residentes na Spaan na época do estudo, participaram dos testes, 33 idosos de ambos os sexos com mais de 60 anos (de 64 a 93 anos; 21 mulheres), considerados aptos a participar e que concordaram em fazer o TC6M (AMERICAN THORACIC SOCIETY,
2002). Além daqueles que se negaram, foram excluídos os idosos que apresentaram contraindicações ao teste, como - angina instável, infarto do miocárdio, frequência cardíaca de repouso de mais de 120 batimentos por minuto, pressão sanguínea sistólica de mais de 180 mmHg e pressão arterial diastólica superior a $100 \mathrm{mmHg}$, durante o mês anterior ao teste (AMERICAN THORACIC SOCIETY, 2002). Também foram excluídos os indivíduos incapazes de realizar a avaliação por contraindicação para atividade física por problemas músculo esqueléticos (fratura em fase de tratamento, risco de luxação), neurológicos, psiquiátricos, vasculares (claudicação intermitente), pulmonares e cardíacos.

O TC6M foi realizado da seguinte forma: ao longo de seis minutos, dois avaliadores, devidamente treinados, orientaram os idosos por meio de frases padronizadas a percorrerem o mais rápido que conseguissem, a maior distância possível, em um percurso de 30 metros, demarcado em um corredor plano e reto dentro da própria instituição, o qual media aproximadamente 100 metros. $\mathrm{O}$ ponto de partida e o final do percurso foram assinalados com cones e o chão marcado com fita adesiva amarela $(6 \mathrm{~cm}$ de largura; $60 \mathrm{~cm}$ de comprimento). $\mathrm{O}$ comprimento do percurso foi assinalado, a cada 3 metros, com pedaços menores e mais estreitos da fita adesiva.

Os participantes foram orientados a interromper o teste caso sentissem extremo cansaço, dispneia, taquicardia, tonturas ou outros sintomas de desconforto. Os testes seriam interrompidos, caso os participantes apresentassem dor no pei- 
to, dispneia, cãibra intensa nos membros inferiores, instabilidade da marcha, sudorese, aparência pálida ou acinzentada.

Os participantes determinaram a sua velocidade da caminhada (RUBIM et al., 2006). Um dos avaliadores foi o responsável por acompanhar o idoso durante todo o percurso do teste. Para evitar a indução do ritmo de caminhada pelo avaliador, esse posicionou-se atrás do idoso, dando-lhe uma das mãos, quando necessário (AMERICAN THORACIC SOCIETY, 2002). Antes do teste (após cinco minutos de repouso sentado) e logo ao final desse, a frequência cardíaca (FC), a pressão arterial sistólica (PAS), a pressão arterial diastólica (PAD), a frequência respiratória (FR), a saturação periférica de oxigênio (oxímetro), o grau de dispneia e de esforço (escala de Borg) foram medidos. A FC foi monitorada, continuamente, durante os testes; o receptor ficava com o avaliador que acompanhava o idoso durante todo o percurso. A PA foi mensurada com um esfigmomanômetro aneroide (fase I e V dos sons de Korotkoff) e a FR foi determinada por meio da contagem do número de incursões respiratórias por minuto, decorrentes do movimento do gradil costal em indivíduos testados.

Os idosos foram classificados quanto ao seu grau de independência funcional em semidependentes ( 85 a 99 pontos) e independentes (100 pontos), de acordo com o seu escore no Índice de Barthel (ARAÚJO et al., 2007). Com esse instrumento, é possível avaliar o nível de independência do indivíduo na realização de dez atividades básicas: comer, tomar banho, vestir e despir, deambular, realizar higiene pessoal, usar os sanitários, controlar esfíncteres, transferir de cadeira para a cama, subir e descer escadas (MAHONEY, BARTHEL, 1965). Um escore acima de 85 pontos significa que o indivíduo apenas necessita de assistência mínima na realização das atividades de vida diária (AVDs).

Os dados receberam tratamento estatístico por meio do software SPSS 13.0 em que o nível de significância $(\alpha)$ de $5 \%$ e, para significância limítrofe os níveis de compreendidos entre 5 e $10 \%$ como critério de decisão. A distribuição dos dados foi verificada com o teste de Shapiro Wilk.

Foi comparada a média da distância máxima percorrida (DMP) do TC6M em relação ao sexo (masculino e feminino) e grau de dependência funcional (semidependentes e independentes) por meio do teste t-Student para grupos independentes e na relação com a faixa etária, foi implementada a análise de variância One Way, complementada pelo teste de comparações múltiplas de Tukey. Também foi utilizado o teste de correlação de Pearson para avaliar a relação entre a idade e a distância percorrida.

$\mathrm{Na}$ análise referente às variáveis $\mathrm{FC}$, FR, PAS e PAD foi implementado o teste t-Student para dados pareados e o teste de Mann Whitney.

Os princípios éticos foram respeitados de acordo com o estabelecido na resolução 196/96 do CNS-MS e o estudo foi aprovado pelo Comitê de Ética em Pesquisa da PUCRS (17/2006). Todos os participantes assinaram um termo de consentimento livre e esclarecido e uma cópia foi deixada com eles. 
Resultados

A maioria dos idosos eram mulheres $(63,6 \%)$ e tinham entre 71 e 80 anos (média: 77,2 $\pm 6,3$ anos), faixa etária que se mostrou significativamente maior do que as demais (Tabela 1). A distribuição quanto ao grau de dependência (independente $\mathrm{x}$ semidependentes) não apresentou proporções estatisticamente diferentes.
Analisando a distância percorrida no TC6M, observou-se que a média da distância total percorrida foi de 250 metros (Tabela 1), visto que $50 \%$ da amostra percorreu distâncias entre 179 e 315 metros. Não foi detectada diferença estatística significativa $(p>0,05)$ nas distâncias percorridas por mulheres (média: 252,8 m) ou por homens (média: 246,9 m).

Tabela 1 - Distribuição das frequências simples e relativa para o sexo, faixa etária e grau de dependência e, medidas de posição e dispersão para a idade e a distância total percorrida durante o teste da caminhada de seis minutos

\begin{tabular}{lr}
\multicolumn{1}{c}{ Variáveis } & $\begin{array}{c}\text { Valores } \\
\mathrm{n}=33\end{array}$ \\
\hline $\begin{array}{l}\text { Sexo* } \\
\text { feminino }\end{array}$ & $12(36,4)$ \\
masculino & $21(63,6)$ \\
Idade & \\
Média \pm desvio padrão & $77,2 \pm 6,3$ \\
Faixa etária* & \\
de 60 a 70 anos & $19(57,6)$ \\
de 71 a 80 anos & $10(30,3)$ \\
acima de 80 anos & \\
Grau de independência funcional ${ }^{*}$ & $16(48,5)$ \\
independente & $17(51,5)$ \\
semidependente &
\end{tabular}

Quando as médias das distâncias percorridas foram analisadas em relação às faixas etárias, o teste de análise de variância não apontou diferença esta- tisticamente significativa. Igualmente, quando utilizado o teste e a correlação de Pearson, não foi detectada correlação entre a DMP no TC6M e a idade (Figura 1). 
Figura 1 - Gráfico de dispersão entre a idade e a distância total percorrida (m) no teste de caminhada de seis minutos

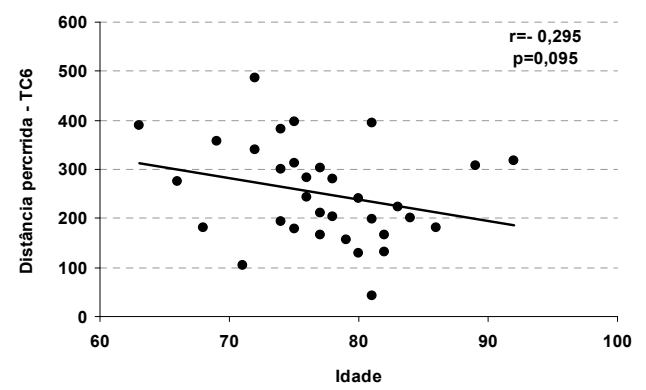

Apesar de os idosos independentes (média: 280,9 m) terem percorrido, em média, quase 60 metros a mais que os semidependentes (média: 222,2 m), não avaliou-se como significativa a diferença encontrada (Figura 2).

A média final da FC mostrou-se, significativamente, maior $(\mathrm{p}<0,001)$ do que a média inicial, segundo o teste $\mathrm{t}$ -Student. A maioria dos idosos (93,9\%) apresentou valores finais superiores (Figura 3). A FR final também foi identificada como mais elevada, diferença estatística significativa determinada por meio do teste de Mann Whitney, no qual os valores finais apresentaram-se superiores em 69,6\% dos idosos (Figura 3).

Figura 2 - Média da distância total percorrida no teste de caminhada de seis minutos, segundo o grau de dependência

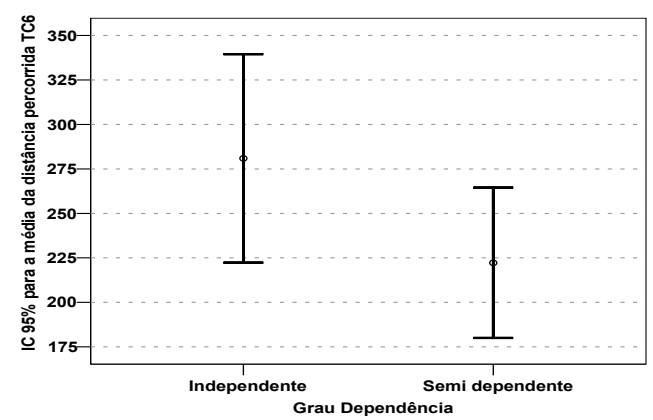

Figura 3 - Análise da frequência cardíaca (FC), frequência respiratória (FR), pressão arterial sistólica (PAS), pressão arterial diastólica (PAD), antes e depois do teste de caminhada de seis minutos

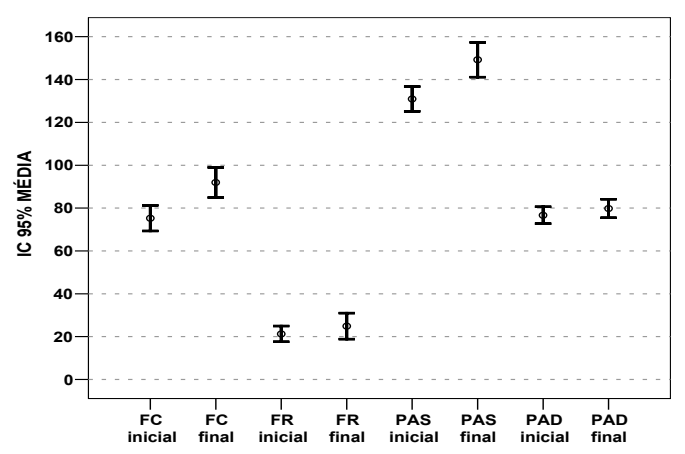

Para a PAS, o teste t-Student indicou que, ao final, essa mostrava-se estatisticamente mais elevada (diferença de 19 mmHg) em 90,9\% dos idosos (Figura 3). A média final da PAD mostrou-se superior a média inicial (diferença de $4 \mathrm{mmHg}$ ), e, segundo o teste t-Student, foi detectada significância limítrofe (Figura 3).

\section{Discussão}

Os resultados encontrados mostraram que a maioria dos idosos da ILPI estudada é composta por mulheres acima de 70 anos, o que condiz com a literatura (OLIVEIRA et al., 2006). Estudos mostram que idosos mais jovens estão em suas casas ou em casas de familiares e, idosos com faixa etária maior acabam indo para instituições, às vezes por opção própria, mas, na maioria das vezes é, ainda, por opção familiar (OLIVEIRA et al., 2006; PEROSA; PAZ; AIRES, 2006).

Em relação ao grau de independência funcional, os grupos foram ho- 
mogêneos, em termos de distribuição, inclusive por sexo, idade e tempo de institucionalização. Aqueles classificados como semidependentes deambulavam sem auxílio de terceiros e precisavam de pouco auxílio nas AVDs, o que pode explicar a ausência de diferença significativa entre estes, no que tange ao desempenho no TC6M.

A média da DMP no TC6M foi de 250 metros, uma distância baixa, quando comparada com a média de 450 metros para idosos com evidência de cardiopatia e de 500 metros para idosos saudáveis, conforme dados encontrados na literatura (ARAÚJO et al., 2006; BRITTO; SOUSA, 2006). Essa discrepância, provavelmente, deve-se ao sedentarismo, resultante da institucionalização dos idosos estudados, posto que nenhum desses praticava atividade física regular, além de ficarem circunscritos aos limites físicos da ILPI. Dentre esses, apenas três mulheres saíam sozinhas da instituição para fazer compras no mercado mais próximo, distante cerca de 200 metros apenas.

Ao buscarmos na literatura estudos com valores para o TC6M em idosos institucionalizados, surpreendeu-nos, por exemplo, a média encontrada por Souza e colaboradores $(429 \pm 70,6 \mathrm{~m})$ em idosos institucionalizados de Florianópolis (SOUZA et al., 2011). Infelizmente, os autores não forneceram a idade dos seis homens e das seis mulheres que participaram do estudo, assim como não apresentaram a média da distância total percorrida de acordo com o sexo, ou a comparação entre estes. Estes aspectos, além do reduzido tamanho amostral, impedem uma comparação adequada entre os nossos dados e os dos autores mencionados.

Um estudo multicêntrico desenvolvido em seis ILPIs brasileiras, com um total de 78 idosos (GONÇALVES et al., 2010), cuja a distribuição da amostra em termos de sexo $(56,4 \%$ de mulheres $)$ e de média de idade $(77,37 \pm 7,87$ anos $)$ foram semelhantes às do presente estudo, a DMP encontrada pelos autores $(263,82 \pm 117,08 \mathrm{~m})$ também foi parecida à encontrada $(250,7 \pm 99,7 \mathrm{~m})$. Os autores, usando valores percentis, classificaram em três categorias a resistência aeróbia da amostra (fraca, regular e boa), uma vez que as médias encontradas para os homens e para as mulheres, no presente trabalho, e também a média geral, encaixaram-se na categoria regular. Em estudo publicado recentemente por Benedetti et al. (2014), os dados confirmam os de Gonçalves et al. (2010), presentes também neste estudo, inclusive a classificação da DMP como regular. Por conseguinte, os resultados indicam que os idosos em pesquisa poderiam beneficiar-se da instituição de um programa de atividade física regular para melhorar o seu condicionamento, utilizando o próprio TC6M como medida do seu progresso, e, também, como meio de estabelecer os níveis de segurança dos exercícios, posto que durante as testagens não surgiram quaisquer intercorrências.

$\mathrm{O}$ aumento significativo dos sinais vitais ao final do teste condiz com a literatura (AMERICAN THORACIC SOCIETY, 2002; BRITO et al., 2002; FESTSCHRIFT, 2006). Nesse sentido, era esperado que a FC aumentasse, linearmente, com a intensidade do esforço 
e, consequentemente, com o consumo de oxigênio, dentro de limites definidos para a prática de esforço físico, que neste caso foi submáximo (BRITO et al., 2002). Após a prática de atividade física, espera-se que o esforço ventilatório aumente (BRITO et al., 2002), o que justifica o aumento encontrado na FR, assim como também é esperado que a PAS aumente com a intensidade crescente do trabalho aplicado, em geral, até $220 \mathrm{mmHg}$ (FESTSCHRIFT, 2006) e que a PAD mantenha-se constante ou oscile cerca de $10 \mathrm{mmHg}$ (FESTSCHRIFT, 2006), o que corrobora com os achados, desta pesquisa, relativos à pressão arterial.

Ainda mais relevante foi o resultado de que em nenhum dos participantes foi detectada hipertensão reativa ao esforço, a qual é conceituada como o achado de valores de $\mathrm{PAS}>220 \mathrm{mmHg}$ e/ou elevação de $15 \mathrm{mmHg}$ ou mais de $\mathrm{PAD}$, partindo de valores normais de pressão em repouso (BRITO et al., 2002; FESTSCHRIFT, 2006). Esses dados demonstram a segurança de aplicar o teste a partir de uma seleção clínica criteriosa, baseada nas indicações e contraindicações relativas ao próprio teste, sem necessidade de exames mais caros, como testes ergométricos (AMERICAN THORACIC SOCIETY, 2002).

A certeza da segurança dos testes foi confirmada mais tarde, quando parte do mesmo grupo de idosos participou de uma intervenção, envolvendo treinamento na esteira e com pesos, durante três meses, sem qualquer tipo de intercorrência ou evento adverso, apresentando, ao final da intervenção, aumento significativo na DMP. ${ }^{1}$
Tanto homens, quanto mulheres percorreram distâncias, estatisticamente, semelhantes, apesar de a média alcançada pelas mulheres ter sido mais elevada do que a dos homens, confirmando resultados encontrados por outros autores, os quais apontam para a igualdade entre os sexos, desde que resguardadas as mesmas condições de saúde (TORAMAN; AYCEMAN, 2005).

A ausência de relação da DMP com a idade e com o grau de independência funcional, entretanto, não condiz com o que se esperaria, uma vez que, com o avanço da idade, ocorrem alterações funcionais características do envelhecimento normal, as quais resultam na diminuição da massa magra, da força muscular, do nível de atividade física (SEBASTIÃO et al., 2009), bem como da capacidade para realizar AVDs (DUARTE et al., 2007). Logo, o esperado seria uma relação negativa da idade com a DMP e com o grau de independência funcional, ou seja, quanto maior a idade, menor a DMP e a independência funcional do indivíduo.

A direção da relação entre a idade e a $\operatorname{DMP}(r=-0,295)$ condiz com o esperado, porém a correlação não atingiu significância $(p=0,095)$, possivelmente em função de uma das limitações deste estudo, o seu tamanho amostral, pequeno demais para que se possa fazer inferências para a população. Não obstante, os achados confirmam duas coisas: (1) o maior sedentarismo dos idosos institucionalizados impacta no seu condicionamento e (2) seguindo as orientações relativas à aplicação do TC6M, este provou ser seguro para ser utilizado fora de uma instituição hospitalar ou 
clínica. Com isso, os limites seguros para a prática de exercícios entre idosos institucionalizados podem ser estabelecidos, sem grandes custos, para as ILPI, e os benefícios das atividades desenvolvidas podem ser, objetivamente, acompanhados por meio do desempenho no TC6M. Essa segurança aconteceu independente do sexo ou da idade, demonstrando que esses homens e mulheres com até 93 anos poderiam se engajar em exercícios, o que, inclusive, poderia diminuir o risco de quedas (PIMENTEL; SCHEICHER, 2009), o qual é, significativamente, mais alto em idosos institucionalizados (CAMPOS et al., 2010).

\section{Conclusões}

Conclui-se que idosos institucionalizados de ambos os sexos conseguiram executar, em segurança, o TC6M, porém percorreram distâncias bem menores do que o esperado, apresentando, portanto, condicionamento físico limitado. Dada a carência de publicações referente ao uso do TC6M em idosos institucionalizados na literatura nacional e internacional, sugere-se o desenvolvimento de novos estudos que confirmem ou refutem os resultados aqui apresentados.
Six-minute walk test in institutionalized elderly: values, applicability and correlations

\section{Abstract}

A sedentary lifestyle, among other factors, leads to increased prevalence of physical limitations and comorbidities in institutionalized elderly. Thus, determining means and / or instruments to aid the detection and prevention of both is essential to ensure better health and quality of life for them. Among the many instruments used for this, the six-minute walk test (6MWT) objectively assesses the degree of physical capacity in individuals with limited physical conditioning. Therefore, this study was developed with the following objectives: (1) to determine the maximum distance walked by the institutionalized elderly in the 6MWT, (2) to verify the relationship between distance walked with gender, age and degree of functional independence and (3) to determine the safety of its use for this population. Participated in this cross-sectional study 33 elderly subjects ( 12 women) living in a long-stay institution, classified as independent and semi-independent (Barthel Index), and able to perform the 6MWT. Vital signs were measured before and after the test. The age of participants ranged from 71 to 80 years. The average maximum distance walked by the elderly was 250 meters, far below the average of 450 meters found in elderly subjects with evidence of heart disease, described in the literature. There was no significant difference in the comparisons of mean distance walked in terms of sex, age or degree of functional independence. It is concluded that institutionalized elderly walked shorter distances than expected, thus presenting limited physical conditioning. The Six-Minute Walk Test has good applicability for this public.

Keywords: Long-stay Institutions for the Elderly. Elderly. Aging. Human Physical Conditioning. 


\section{Nota}

1 Dados disponibilizados pela autora.

\section{Referências}

AMERICAN THORACIC SOCIETY. Statement: guidelines for the six-minute walk test. American Journal of Respiratory and Critical Care Medicine, New York, v. 166, n. 1, p. 111-117, jul. 2002.

ARAÚJO, C. O. et al. Diferentes padronizações do teste de caminhada de seis minutos para mensuração da capacidade de exercício de idosos com e sem cardiopatia clinicamente evidente. Arquivos Brasileiros de Cardiologia, São Paulo, v. 86, n. 3, p. 342-348, mar. 2006.

ARAÚJO, F.; RIBEIRO, J. L. P.; OLIVEIRA, A.; PINTO, C. Validação do Índice de Barthel numa amostra de idosos não institucionalizados. Revista Portuguesa de Saúde Pública, local de publicação, v. 25, n. 2, p. 59-66, jul./ dez. 2007.

BENEDETTI, T. R. B.; MAZO, G. Z.; GONÇALVES, L. H. T. Bateria de testes da AAHPERD: adaptação para idosos institucionalizados. Rev Bras Cineantropom Desempenho Hum., local de publicação, v. 16, n. 1, p. 1-14, 2014.

BRASIL. Instituto Brasileiro de Geografia e Estatística. Ministério do Planejamento, Orçamento e Gestão. Síntese de indicadores sociais: uma análise das condições de vida da população brasileira. Estudos e Pesquisa: Informação Demográfica e Socioeconômica, Rio de Janeiro, n. 29, 2012.

BRITO, F. S. et al. II Diretrizes da Sociedade Brasileira de Cardiologia Sobre Teste Ergométrico. Arquivos Brasileiros de Cardiologia, São Paulo, v. 78, supl. 2, p. 1-17, 2002. Disponível em: http://<www.scielo.br/ scielo.php?script=sci_arttext\&pid=S0066-782X2002000800001>. Acesso em: 19 maio 2013.
BRITTO, R. R., SOUSA, L. A. P. Teste de caminhada de seis minutos: uma normatização brasileira. Fisioterapia em Movimento, Curitiba, v. 19, n. 4, p. 49-54, out./ dez. 2006.

CAMPOS, L. M.; REHDER, S.; ROSADO, M. Relatório global da organização mundial saúde sobre prevenção de quedas na velhice. São Paulo: Secretaria de Estado da Saúde, 2010.

CHAIMOWICZ, F. A saúde dos idosos às do século XXI: problemas, projeções e alternativas. Revista de Saúde Pública, São Paulo, v. 31, n. 2, p. 184-200, abr. 1997.

CREUTZBERG, M. A instituição de longa permanência para idosos e sua relação com o sistema societal: uma análise na perspectiva da teoria de sistemas de Niklas Luhmann. 2005. Tese (Doutorado em Gerontologia Biomédica) - Instituto de Geriatria e Gerontologia da Pontifícia Universidade Católica do Rio Grande do Sul, Porto Alegre, 2005.

DUARTE, Y. A. O.; ANDRADE, C. L.; LEBRÃO, M. L. O Índice Katz na avaliação da funcionalidade dos idosos. Revista da Escola de Enfermagem - USP, São Paulo, v. 41, n. 2, p. 317-325, ago. 2007.

FESTSCHRIFT, B. Exercise is good for your blood pressure: effects os endurance training and resistance training. Clinical and Experimental Pharmacology and Physiology, local de publicação, v. 33, n. 9, p. 853-856, set. 2006.

GONÇALVES, et al. O idoso institucionalizado: avaliação da capacidade funcional e aptidão física. Cad. Saúde Pública, Rio de Janeiro, v. 26, n. 9, p. 1738-1746, set. 2010.

GUCCIONE, A. A. Fisioterapia geriátrica. 2. ed. São Paulo: Guanabara, 2002. p. 470.

IORIS, M. N. Fisioterapia no abrigo Bezerra de Menezes: uma abordagem da fisioterapia na terceira idade. Belo Horizonte, 2002. Disponível em: http://www.redadultosmayores. com.ar/buscador/files/SALUD056.pdf. Acesso em: 19 maio 2013. 
MAHONEY, F. I.; BARTHEL, D. W. Functional Evaluation: the Barthel Index. Maryland State Medical Journal, v. 14, p. 56-61, 1965.

OLIVEIRA, C. R. M.; SOUZA C. S.; FREITAS, M. Idosos e família: asilo ou casa. Revista de Psicologia, Fortaleza, 2006. Disponível em: http:/www.psicologia.pt/artigos/textos/ A0281.pdf. 2006. Acesso em: 19 maio 2013.

PAPALÉO, N. M.; PONTE, J. R. Envelhecimento: desafio da transição do século. In: PAPALÉO N. M. Gerontologia: a velhice e o envelhecimento em visão globalizada. São Paulo: Atheneu, 2005. p. 3-12.

PEROSA, C. T.; PAZ, A. A.; AIRES, M. O. Grau de dependência e características de pessoas idosas institucionalizadas. Revista Brasileira de Ciências do Envelhecimento Humano, Passo Fundo, v. 3, n. 2, p. 79-91, out./dez. 2006.

PIMENTEL, R.; SCHEICHER, M. E. Comparação do risco de queda em idosos sedentários e ativos por meio da escala de equilíbrio de Berg. Fisioterapia \& Pesquisa, v. 16, n. 1, p. 6-10, 2009.

RUBIM, V. S. M. et al. Valor prognóstico do teste de caminhada de seis minutos na insuficiência cardíaca. Arquivo Brasileiro de Cardiologia, São Paulo, v. 86, n. 2, p. 120125, fev. 2006.

SEBASTIÃO, E. et al. Atividade física, qualidade de vida e medicamentos em idosos: diferenças entre idade e gênero. Revista Brasileira de Cineantropometria e Desempenho Humano, Florianópolis, v. 11, n. 2, p. 210-216, jul./ago. 2009.

SOLWAY, S.; BROOKS, D.; LACASSE, Y.; THOMAS, S. A qualitative systematic overview of the measurement properties of functional walk tests used in the cardiorespiratory domain. Chest, Chicago, v. 119, n. 1, p. 256-270, jan. 2001.

SOUZA, P. D.; BENEDETTI, T. R. B.; BORGES, L. J.; MAZO, G. Z.; GONÇALVES, L. $\mathrm{H}$. T. Aptidão funcional de idosos residentes em uma instituição de longa permanência.
Rev. Bras. Geriatr. Gerontol, Rio de Janeiro, v. 14, n. 1, p. 7-16, 2011.

SUN, F.; NORMAN, I. J.; WHILE, A. E. Physical activity in older people: a systematic review. BMC Public Health, v. 13, p. 449, 2013. doi: 10.1186/1471-2458-13-449.

TORAMAN, N. F.; AYCEMAN, N. A. Effects of six weeks of detraining on retention of functional fitness of old people after nine weeks of multicomponent training. British Journal Sports Medicine, London, v. 39, n. 8, p. 565-568, dez. 2005. 